Braz J Med Biol Res, October 2012, Volume 45(10) 921-928

doi: 10.1590/S0100-879X2012007500106

Effect of different durations of overdistention on rat bladder function and morphology

Xiao-Dong Jin, Min Cao, Xie-Lai Zhou, Hong-Ping Yin, Zhao-Dian Chen, Ning Xu and Hai Jiang

The Brazilian Journal of Medical and Biological Research is partially financed by

\section{惡CNPq}

da Ciência e Tecnologia

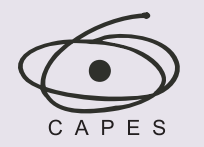

Ministério da Educação
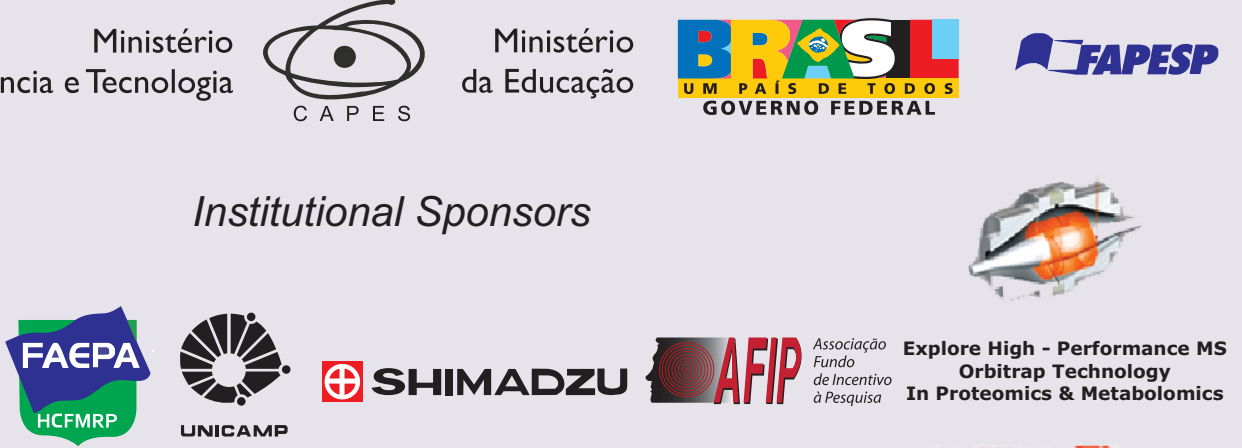

Institutional Sponsors

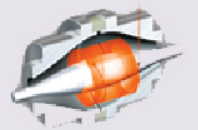
UNICAMP
Ф SHIMADZU

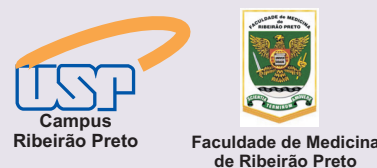
de Ribeirão Preto
de 


\title{
Effect of different durations of overdistention on rat bladder function and morphology
}

\author{
Xiao-Dong Jin ${ }^{1}$, Min Cao ${ }^{1}$, Xie-Lai Zhou ${ }^{2}$, Hong-Ping Yin ${ }^{2}$, Zhao-Dian Chen ${ }^{1}$, \\ Ning $\mathrm{Xu}^{3}$ and Hai Jiang ${ }^{1}$ \\ ${ }^{1}$ Department of Urology, The First Affiliated Hospital, Zhejiang University School of Medicine, Hangzhou, China \\ ${ }^{2}$ College of Medicine, Hangzhou Normal University, Hangzhou, China \\ ${ }^{3}$ College of Pharmaceutical Science, Zhejiang University of Technology, Hangzhou, China
}

\begin{abstract}
We investigated the contribution of the duration of overdistention (DOD) to rat bladder function and morphology and explored its possible molecular mechanisms. Bladder overdistention was induced in male Sprague-Dawley rats (200-250 g) by an infusion of saline. Forty rats were divided into 5 groups submitted to different DOD, i.e., 1, 2, 4, and $8 \mathrm{~h}$, and control. Bladder function was evaluated by cystometry. Morphological changes were observed by light and transmission electron microscopy. Compared to control $\left(44.567 \pm 3.472 \mathrm{cmH}_{2} \mathrm{O}\right)$, the maximum detrusor pressure of groups with 2-, 4- and 8-h DOD decreased significantly (means \pm SEM): $32.774 \pm 3.726,31.321 \pm 2.847$, and $29.238 \pm 3.724 \mathrm{cmH}_{2} \mathrm{O}$. With the increase of DOD, inflammatory infiltration and impairment of ultrastructure were more obvious in bladder tissue. Compared to control (1.90 \pm 0.77$)$, the apoptotic indexes of groups with 1-, 2-, 4-, and 8-h DOD increased significantly $(6.47 \pm 2.10,10.66 \pm 1.97,13.91 \pm 2.69$, and $18.33 \pm 3.28 \%)$. Compared to control $(0.147 \pm 0.031 / 0.234 \pm 0.038$ caspase $3 / \beta$-actin and Bax/Bcl-2 ratios), both caspase $3 / \beta$-actin and Bax/ $\mathrm{Bcl}-2$ ratios of 1-, 2-, 4-, and 8-h DOD increased significantly $(0.292 \pm 0.037 / 0.508 \pm 0.174,0.723 \pm 0.173 / 1.745 \pm 0.471,1.104$ $\pm 0.245 / 4.000 \pm 1.048$, and $1.345 \pm 0.409 / 8.398 \pm 3.332$ ). DOD plays an important role in impairment of vesical function and structure. With DOD, pro-apoptotic factors increase and anti-apoptotic factors decrease, possibly contributing to the functional deterioration and morphological changes of the bladder.
\end{abstract}

Key words: Acute urinary retention; Bladder overdistention; Urodynamics; Ultrastructure; Apoptosis; Rat

\section{Introduction}

Acute urinary retention (AUR) is a common complication in men with benign prostatic hyperplasia and/or low urinary tract symptoms $(1,2)$. A 2-year study of finasteride showed that approximately $3 \%$ of placebo-treated subjects suffered an episode of AUR (3). As an indication for prostatectomy, AUR accounts for $24-42 \%$ of patients undergoing prostatectomy (4).

Traditionally, AUR has been thought to simply produce stretch damage of the detrusor muscle and other bladder microstructures, resulting in reduced contractility of the detrusor muscle. Animal studies have indicated that the biochemical and metabolic status of the bladder is altered after overdistention $(5,6)$. Ischemia-reperfusion injury after overdistention plays an important role in prolonged bladder dysfunction $(7,8)$. Overdistention induces increased pressure in the bladder and reduction in blood flow to bladder tissue, resulting in partial ischemia and hypoxia. After decompression, the blood supply recovers and oxygen free radicals are generated, ultimately leading to bladder dysfunction.

The structural changes of the bladder occurring with AUR time have been confirmed by Gabella and Uvelius (9). However, ischemia-reperfusion injury was not considered and bladder function was not investigated simultaneously in their study. Furthermore, few reports are available about the role of apoptosis and related factors in bladder dysfunction after AUR.

In the present study, we created a new overdistention rat model and determined the morphological and functional changes of the bladder with duration of the condition. We also observed the changes of apoptotic rates and apoptosisrelated factors in bladder tissue after different durations of overdistention (DOD). We attempted to determine whether different DOD could result in different severities of blad-

Correspondence: Hai Jiang, Department of Urology, The First Affiliated Hospital, Zhejiang University School of Medicine, 79 Qingchun Road, Hangzhou 310003, China. Fax: +86-571-8723-6594. E-mail: kingshoulder@163.com

Received June 1, 2011. Accepted May 28, 2012. Available online June 29, 2012. Published September 3, 2012. 
der impairment, to determine the relationship between morphological and functional changes, and to prove the important role of apoptosis and its related factors in the bladder dysfunction occurring after overdistention.

\section{Material and Methods}

\section{Animal model}

All animal care and experimental protocols were in accordance with the guidelines of $\mathrm{NIH}$ regarding the care and use of animals for experimental procedures, and were approved by the animal laboratory of Zhejiang University. Forty male Sprague-Dawley rats (200-250 g, 3-4 months of age) were anesthetized with urethane $(1.0 \mathrm{~g} /$ $\mathrm{kg}, i p)$ and allocated to five groups of 8 rats each: shamoperated control, DOD for $1 \mathrm{~h}$, DOD for $2 \mathrm{~h}$, DOD for 4 $\mathrm{h}$, and DOD for $8 \mathrm{~h}$. The bladder was exposed through a lower abdominal midline incision. After emptying the bladder, we ligated the foreskin with a 3-0 silk thread. A 24-G catheter was inserted into the apex of the bladder dome and connected to an infusion pump and $0.9 \%$ saline was infused $(0.1 \mathrm{~mL} / \mathrm{min})$ at $37^{\circ} \mathrm{C}$ up to a total volume of $1.0 \mathrm{~mL}$, which was approximately twice the mean bladder volume of $0.5 \mathrm{~mL}$. The status of overdistention was kept for 1, 2, 4 , and $8 \mathrm{~h}$, respectively. In the control group, the bladder was exposed and punctured as in other groups but without saline infusion. A cystometric study was performed after overdistention relief for $1 \mathrm{~h}$.

\section{Cystometric study}

After emptying the bladder, we inserted a 24-G catheter into the apex of the bladder dome. The catheter was connected to an infusion pump and $0.9 \%$ saline was infused $(0.1 \mathrm{~mL} / \mathrm{min})$ at $37^{\circ} \mathrm{C}$ until micturition was detected. The catheter was also connected to an external pressure transducer for the measurement of intravesical pressure. Data were recorded on a personal computer via a bridge amplifier. The following parameters were recorded: intravesical pressure at the beginning and end of filling, bladder capacity, and maximum detrusor pressure during voiding. Detrusor overactivity and detrusor acontraction are reported according to the definition of the International Continence Society (10).

\section{Contractile responses assayed in vitro}

After cystometry, rats were sacrificed and the bladder was removed at the level of the bilateral urethral orifices. Approximately 8-mm long and 2-mm wide detrusor strips were prepared from the bladder body and placed in an organ bath containing Krebs-Hanseleit solution $\left(37^{\circ} \mathrm{C}\right.$, $\mathrm{pH} 7.4$ ) continuously infused with $95 \% \mathrm{O}_{2}$ and $5 \% \mathrm{CO}_{2}$. After preparation, one end of each prepared strip was connected to a muscle holder and the other end to an FDT 10 force displacement transducer (Biopac, USA) with a silk thread. Before constructing concentration-response curves, muscle strips were stretched to the length of optimal force development and then equilibrated for $60 \mathrm{~min}$. During this period, the Krebs-Hanseleit solution was replaced every $15 \mathrm{~min}$. Electrical-field stimulation (EFS) was applied to the strips at 2, 4, 8, 16, and $32 \mathrm{~Hz}$ for $20 \mathrm{~s}$ with 1-ms, 80-V pulses using an S88 stimulator (Grass Instruments, USA). Next, the maximum contractile responses to $0.3,1.0,3.0$, 10 , and $30 \mu \mathrm{m}$ carbachol and $120 \mathrm{mM}$ potassium chloride were determined. The strips were washed three times with fresh buffer between studies with different drugs. The data were recorded using the MP 100 software (Biopac).

\section{Morphological examination}

To assess inflammatory infiltrates, sections were stained with hematoxylin and eosin and examined by light microscopy. To observe the ultrastructure of the bladder tissues, sections were stained with uranyl acetate and lead citrate and then examined by transmission electron microscopy.

\section{Terminal deoxynucleotidyl transferase-mediated nick-end labeling (TUNEL) assay}

Apoptosis was detected by the TUNEL assay. Formalinfixed, paraffin wax-embedded tissue sections were deparaffinized and stained by the TUNEL-avidin-biotin-complex method. Ten high-power (400X) fields were randomly selected in each section. We selected different numbers of fields according to the thickness of different layers: 2 in the mucosa, 2 in the submucosa, 4 in the detrusor, and 2 in the serosa. The number of positive cells and total cells was recorded and analyzed. The apoptotic index was calculated as number of positive cells/number of total cells $\times 100 \%$.

\section{Western blotting}

To measure the expression of $\mathrm{Bcl}-2$, Bax, and caspase-3 at the protein level, bladder samples were quickly frozen in liquid nitrogen, and stored at $-70^{\circ} \mathrm{C}$ for protein isolation. The tissues were homogenized with a pre-chilled mortar and pestle in extraction buffer. After complete homogenization, the sample was kept at $4^{\circ} \mathrm{C}$ for $30 \mathrm{~min}$, centrifuged at $12,000 \mathrm{~g}$ for $12 \mathrm{~min}$, and the supernatant was collected. Subsequently, protein concentration was determined by the Bio-Rad protein assay (Bio-Rad Laboratories, USA). The antibodies used for Western blotting analysis were goat polyclonal antispectrin $\alpha$ antibody (Santa Cruz Biotechnology Inc., USA), mouse anti-actin monoclonal antibody, mouse anti-Bcl-2 monoclonal antibody, mouse anti-Bax monoclonal antibody, and rabbit anti-caspase-3 (CPP32) monoclonal antibody (NeoMarkers, USA).

\section{Statistical analysis}

Data are reported as means \pm SEM and the differences between groups were compared by ANOVA. $P<0.05$ was considered to be statistically significant. 


\section{Results}

All rats survived and their data were collected, except for one rat in the group with 8-h overdistention.

\section{Results of the cystometric study}

The bladder capacity and maximum detrusor pressure decreased significantly in groups with 2-, 4-, and 8-h DOD compared to control. Bladder compliance also decreased significantly in the groups with 4- and 8-h overdistention. With the increase of DOD, more rats suffered detrusor overactivity and acontraction (Table 1).

\section{Contractile responses assayed in vitro}

In the groups with 2-, 4- and 8-h overdistention, the maximal contractile responses induced by $32-\mathrm{Hz}$ EFS, 30 $\mu \mathrm{m}$ carbachol, and $120 \mathrm{mM}$ potassium chloride decreased significantly compared to control. The longer the AUR duration, the smaller the maximal contractile response (Figure 1).

\section{Morphological examination}

A few white blood cells (WBCs) infiltrated the mucosa and submucosa of bladder tissues in the 1- and 2-h DOD groups, while few WBCs were seen in muscles and in the external membrane. In the 4-h DOD group, acute inflammation was present in all layers of the bladder and was most obvious in the submucosa. The vessels were hyperemic and dilated, quite a few WBCs infiltrated and the inflammation of the mucosa was also obvious. Even in the muscle, we could see a few WBCs. In the 8-h DOD group, the inflammation was more extensive than in the 4-h DOD group. Hemorrhage was seen in the submucosa and some mucosal cells were sloughed (Figure 2). Although the inflammation in muscle was less than in the other layers, it was more extensive than that of the 4-h DOD group.

\section{Ultrastructural changes of the detrusor}

The ultrastructural changes of muscles and organelles from the 1-and 2-h DOD groups were not obvious compared to the control. In the 4-h DOD group, muscle cells were still intact and lined up in an orderly manner. However, the intercellular gap began to broaden while at the same time some mitochondrial ridges disappeared and vacuoles emerged. In the 8-h DOD group, muscle cells lined up in a disorderly manner and the intercellular space was obviously wider and infiltrated with inflammatory cells. The mitochondria were heavily swollen and their ridges disappeared (Figure 3).

\section{TUNEL assay}

Using the TUNEL method, we found that different groups exhibited varying degrees of positive apoptosis staining. The apoptotic indexes increased with prolonged DOD from $6.47 \pm 2.10 \%$ in the group with $1-\mathrm{h}$ DOD to $18.33 \pm 3.28 \%$ in the group with 8-h DOD (Figure 4).

\section{Apoptosis-related factors}

Western blotting showed that the pro-apoptotic factors Bax and caspase-3 were up-regulated, while the antiapoptotic factor $\mathrm{Bcl}-2$ was down-regulated with the increase of DOD (Figure 5).

\section{Discussion}

In the present study, we improved the overdistention rat model. Some investigators induced overdistention by clamping the distal urethra of rats with a small clip, which could hurt the urethra to some extent and affect the result of cystometry $(7,11)$. Our model was induced by ligating the foreskin, which did not hurt the urethra and ensured correct data for the urodynamic study. According to the literature, the volume of saline infused in the bladder varies in different overdistention models. It was $3 \mathrm{~mL}$ in the rat model used by Saito and Miyagawa (7) and Saito et al. (11) and $1.5 \mathrm{~mL}$ in the model used by Hong et al. (12). So far, no standard infusion volume has been established for the overdistention model. However, many investigators have infused a saline volume twice the bladder volume to induce

Table 1. Cystometric results of rats undergoing different durations of overdistention.

\begin{tabular}{cccccccc}
\hline Group & $\mathrm{N}$ & Capacity $(\mathrm{mL})$ & Compliance $\left(\mathrm{mL} / \mathrm{cmH}_{2} \mathrm{O}\right)$ & Pdet $\left(\mathrm{cmH}_{2} \mathrm{O}\right)$ & DO ratio & DA ratio & Non-micturition ratio \\
\hline $\begin{array}{c}\text { Control } \\
\text { DOD }\end{array}$ & 8 & $0.479 \pm 0.104^{\mathrm{cde}}$ & $0.488 \pm 0.158^{\mathrm{de}}$ & $44.567 \pm 3.742^{\mathrm{cde}}$ & $0 / 8$ & $0 / 8$ & $0 / 8$ \\
1-h & 8 & $0.472 \pm 0.090^{\mathrm{cde}}$ & $0.495 \pm 0.090^{\mathrm{de}}$ & $45.098 \pm 3.649^{\mathrm{cde}}$ & $2 / 8$ & $0 / 8$ & $0 / 8$ \\
2-h & 8 & $0.420 \pm 0.073^{\mathrm{ab}}$ & $0.336 \pm 0.061^{\mathrm{de}}$ & $32.774 \pm 3.726^{\mathrm{abe}}$ & $3 / 8$ & $0 / 8$ & $1 / 8$ \\
4-h & 8 & $0.326 \pm 0.040^{\mathrm{ab}}$ & $0.323 \pm 0.018^{\mathrm{abc}}$ & $31.321 \pm 2.847^{\mathrm{ab}}$ & $5 / 8$ & $2 / 8$ & $2 / 8$ \\
8-h & 7 & $0.296 \pm 0.082^{\mathrm{ab}}$ & $0.259 \pm 0.044^{\mathrm{abc}}$ & $29.238 \pm 3.724^{\mathrm{ab}}$ & $5 / 7$ & $2 / 7$ & $3 / 7$ \\
\hline
\end{tabular}

Pdet $=$ maximum detrusor pressure during voiding; $D O=$ detrusor overactivity; $D A=$ detrusor acontraction. Data were analyzed by ANOVA: aP $<0.05$ vs control group; bP $<0.05$ vs 1-h duration of overdistention (DOD) group; ${ }^{C P}<0.05$ vs $2-h$ DOD group; $\mathrm{d}$ < 0.05 vs 4-h DOD group; ${ }^{\mathrm{eP}}<0.05$ vs 8 -h DOD group. 
the overdistention model. By testing male Sprague-Dawley rats weighing 200-250 g repeatedly, we found that the mean bladder volume was $0.5 \mathrm{~mL}$ and therefore we set $1 \mathrm{~mL}$ as the volume for the overdistention model.

According to the literature, the severity of bladder dysfunction varies at different times after overdistention relief $(7,12)$. Saito et al. (11) measured the contractile responses to carbachol before and at the beginning of overdistention, at the time of overdistention relief, and 30 min after catheterization. They found that the maximum contractile responses of the bladder to carbachol did not change significantly before or at the beginning of overdistention and at the time of overdistention relief. However, the maximum contractile response decreased significantly at $30 \mathrm{~min}$ after catheterization. In order to take the ischemia-reperfusion injury into account, we performed the cystometric tests $1 \mathrm{~h}$ after overdistention relief, while the bladder tissues for the
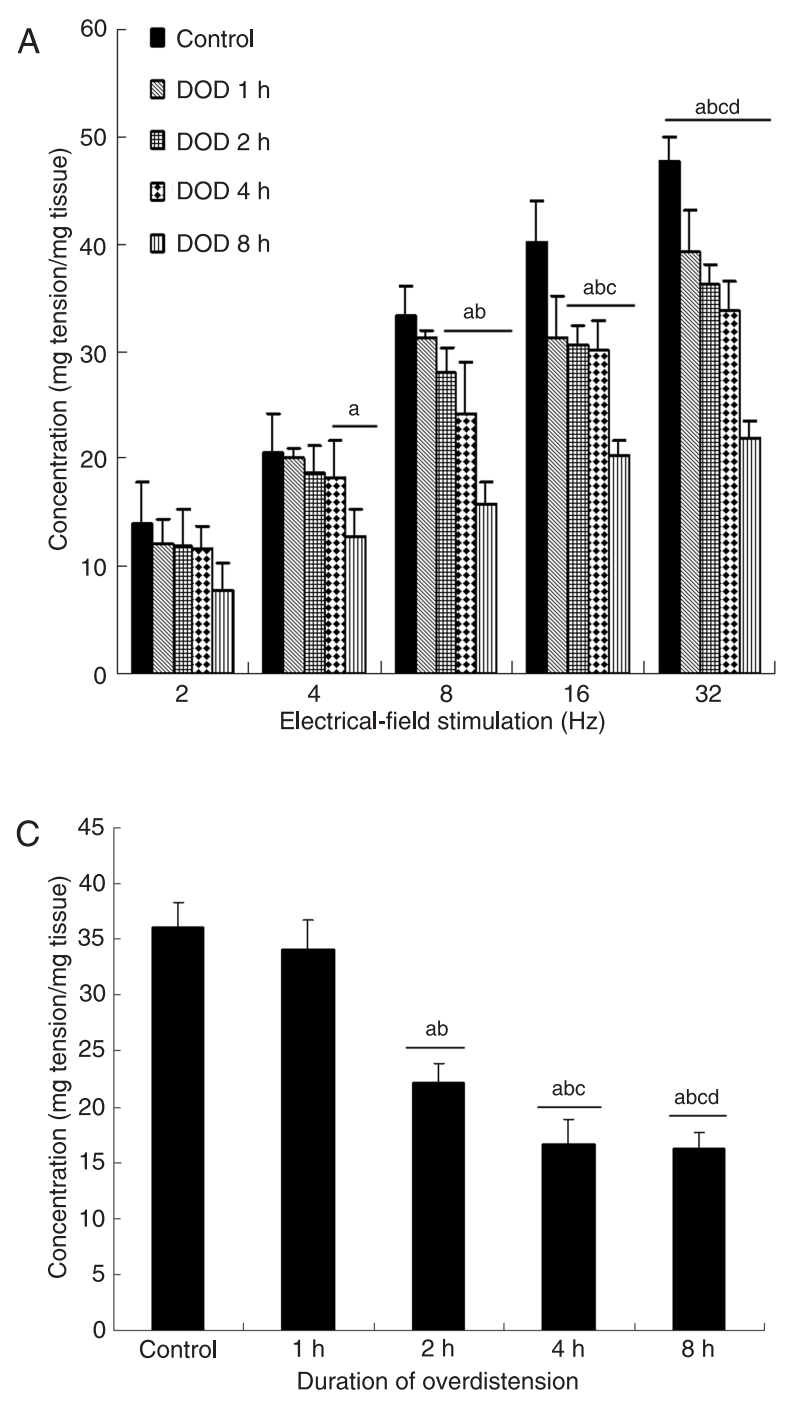

assay of the contractile responses and for pathologic and molecular biology tests were collected immediately after the cystometric tests.

The function of the urinary bladder is to collect and store urine at low intravesical pressures, and then to periodically expel the urine by a coordinated sustained contraction. When AUR occurs, the intravesical pressure is elevated, causing ischemia and anoxia of the detrusor (13). AfterAUR is relieved, the reperfusion injury aggravates the damage to the detrusor (14). As a result, the function of the bladder could be affected by AUR. Our cystometric study showed that overdistention could result in a decrease of maximum detrusor pressure and of bladder capacity and compliance. Detrusor overactivity or detrusor arreflexia could also be the result of overdistention. All these changes became more obvious with the increase of DOD.

We also found that the structural impairment of bladder

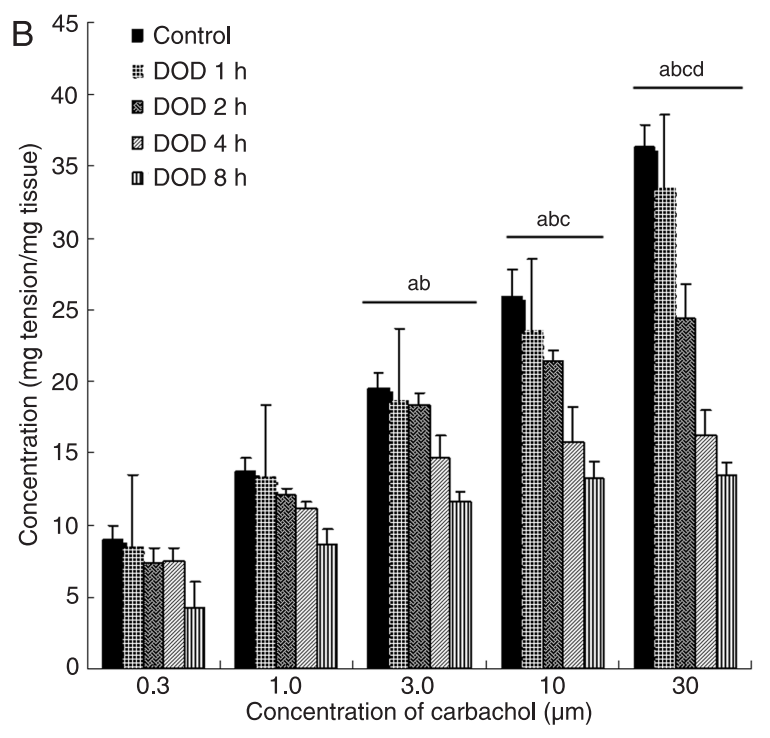

Figure 1. Maximum contractile responses of muscle strips to electrical-field stimulation (EFS) $(A)$, carbachol $(B)$, potassium chloride $(C)$. The maximal contractile responses decreased significantly with increasing duration of overdistention (DOD) when they were induced by 32$\mathrm{Hz}$ EFS, $30 \mu \mathrm{m}$ carbachol and $120 \mathrm{mM}$ potassium chloride. Data were analyzed by ANOVA: aP $<0.05$ vs control group; bP < 0.05 vs 1 -h DOD group; $\mathrm{cP}<0.05$ vs 2-h DOD group; $\mathrm{dP}<0.05$ vs 4 -h DOD group. 

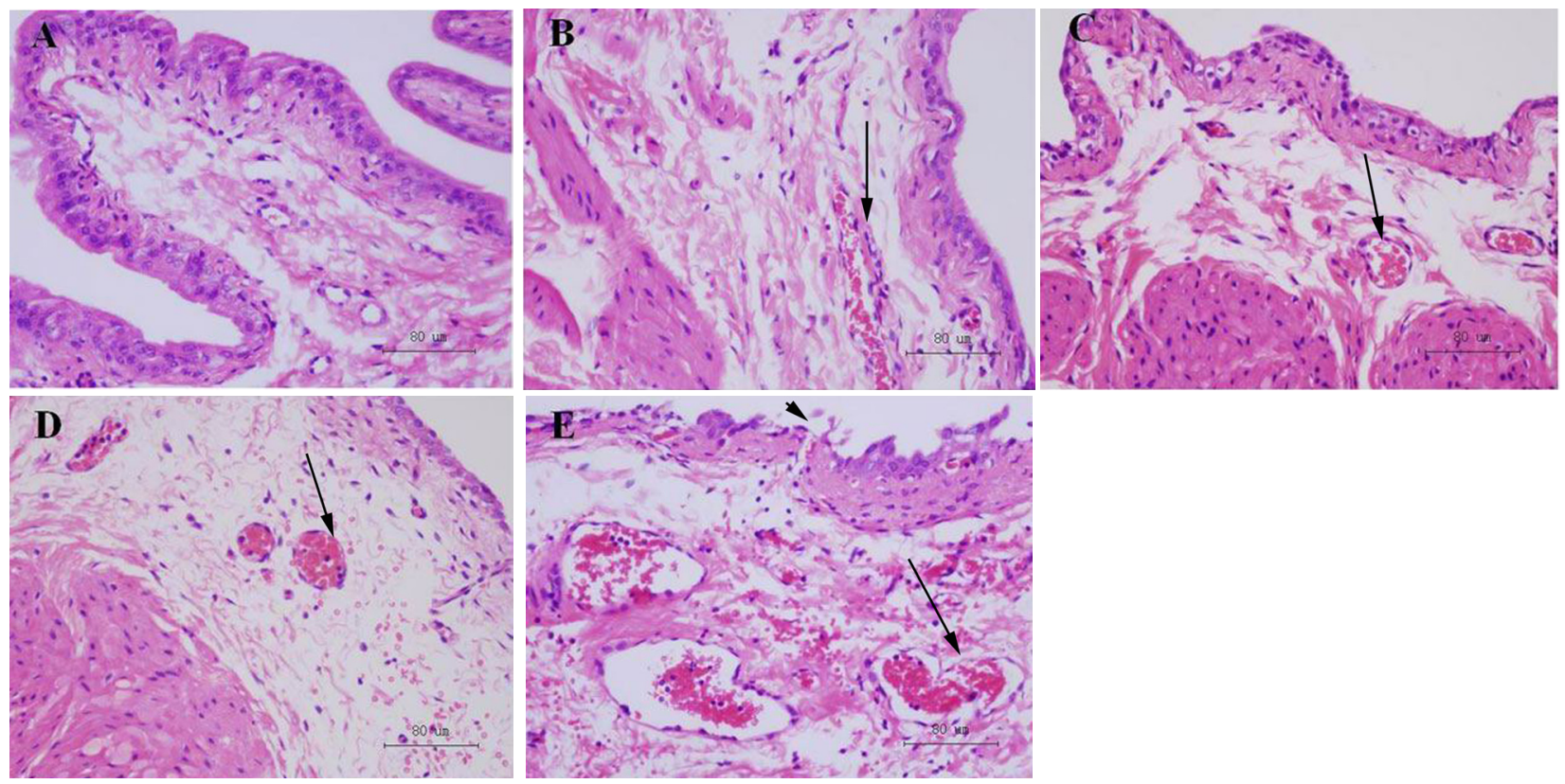

Figure 2. Morphological changes at different durations of overdistention (DOD) determined by microscopy with HE staining. Magnification bars $=80 \mu \mathrm{m}$. $A$, Normal mucosa and submucosa; $B$, hemangiectasis in the submucosa, a few infiltrating inflammatory cells (arrow); $C$, hemangiectasis in the submucosa, more infiltrating inflammatory cells than in the 1-h DOD group (arrow); $D$, hemangiectasis and edema in the submucosa, more red blood cell leakage and infiltrating inflammatory cells (arrow); $E$, more obvious hemangiectasis and edema in the submucosa, obvious red blood cell leakage (arrow) and infiltrating inflammatory cells, some mucosal cells were sloughed (arrowhead).
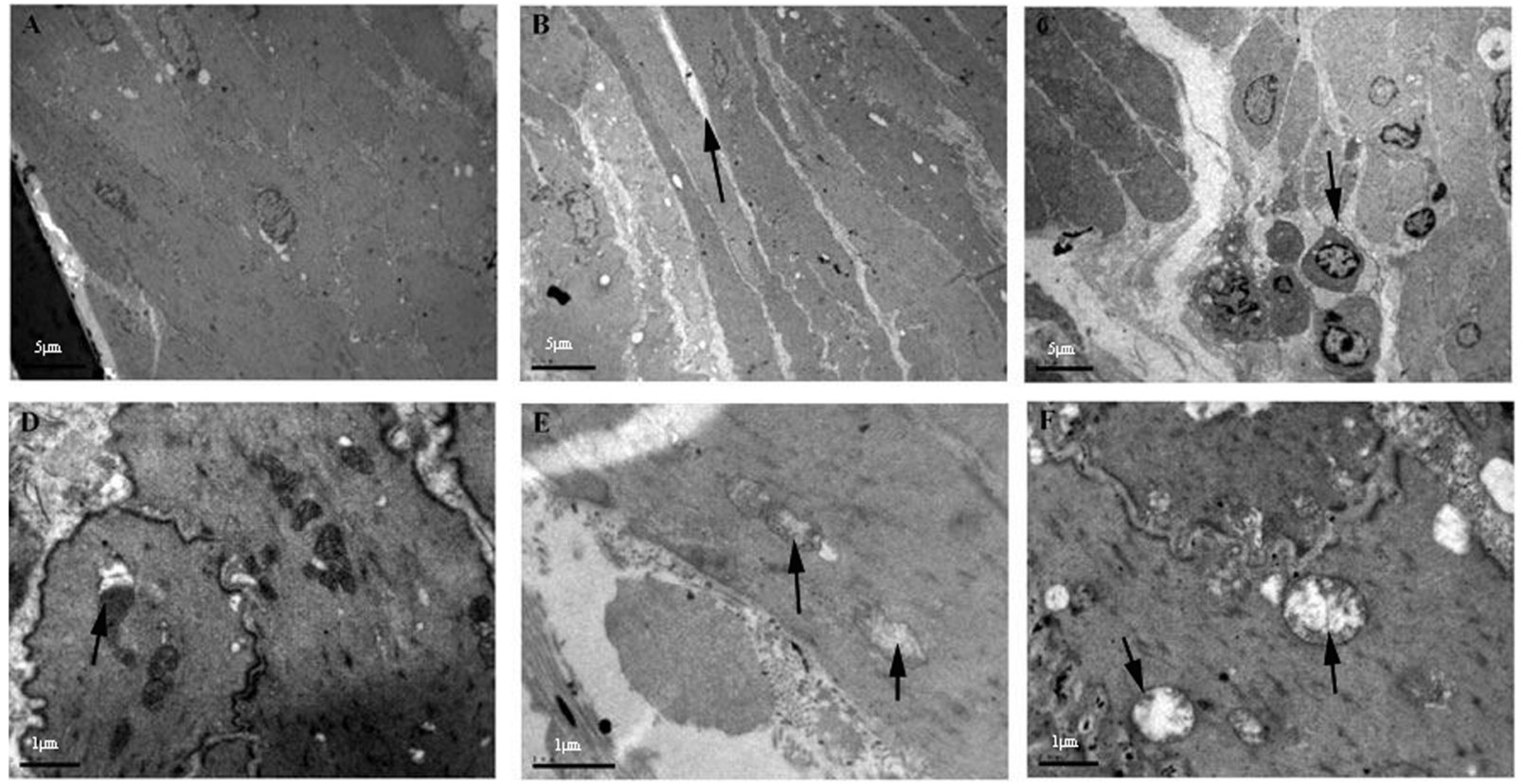

Figure 3. Ultrastructural changes at different durations of overdistention (DOD) observed by transmission electron microscopy. $A$, Control group, normal morphology of myocytes, arranged regularly with intact karyomorphism; $B$, 4-h DOD group, normal morphology of myocytes, arranged regularly with broadening intercellular space (arrow); C, 8-h DOD group, myocytes arranged in a disorderly manner, broadening intercellular space with infiltrating inflammatory cells (arrow); $D$, control group, normal mitochondria with tight intercellular connections (arrow); $E$, 4-h DOD group, ridges of mitochondria disappearing and vacuoles appearing (arrows); $F, 8$-h DOD group, swelling mitochondria without ridges, and more obvious vacuoles (arrows). Magnification bars: $A, B, C=5 \mu \mathrm{m} ; D, E, F=1 \mu \mathrm{m}$. 


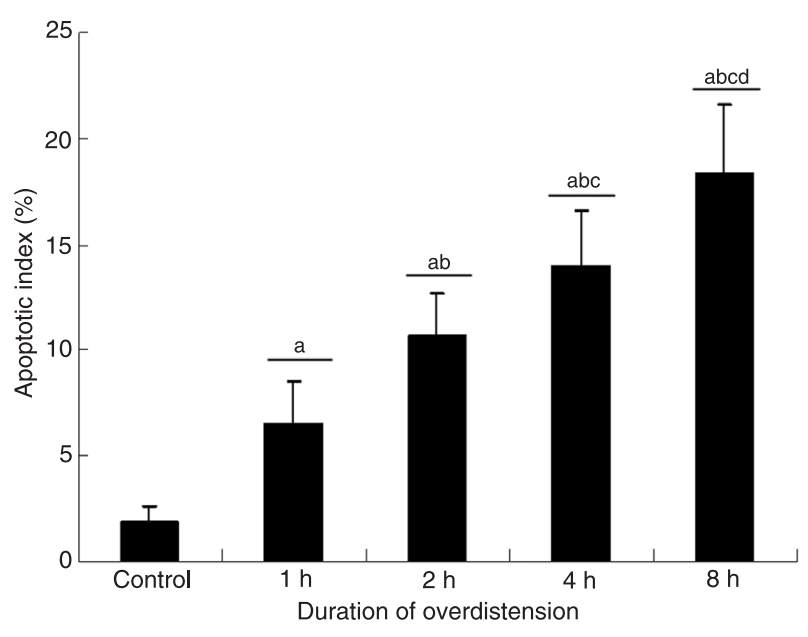

Figure 4. The apoptotic indexes of groups with different durations of overdistention (DOD). The apoptotic indexes increased significantly with increasing DOD. All data were analyzed by ANOVA: ap $<0.05$ vs control group; bP $<0.05$ vs $1-h$ DOD group; $\mathrm{CP}<0.05$ vs 2-h DOD group; ${ }^{\mathrm{d}} \mathrm{P}<0.05$ vs 4 -h DOD group.

tissue worsened with the duration of overdistention, while different layers of bladder tissue showed different severities of structural impairment: the mucosa and submucosa layers were prone to be injured, while the impairment of the muscle layer was usually slight. The possible reasons for these differences are as follows: first, the mucosa and submucosa metabolize actively and are more susceptible to ischemia and anoxia; second, owing to the rich vessels in the mucosa and submucosa, more WBCs exude after overdistention relief, releasing more active oxygen and hydrolytic enzymes that cause more serious tissue injuries; finally, muscle tissue has the strongest antioxidant ability. After observing slight changes in the detrusor by light microscopy, we further investigated it by transmission electron microscopy. As expected, the changes of morphology and organelles were not obvious at 1 and $2 \mathrm{~h}$ after overdistention relief. However, they became more obvious with the increase of DOD, such as a broader intercellular gap, inflammatory cell exudation, and swelling mitochondria with reduced ridges.

We used molecular biology techniques to detect how DOD affected the apoptosis-related factors in the rat bladder. We proved the important role of apoptosis and its related factors in the bladder dysfunction occurring after overdistention. With increased DOD, the expression of anti-apoptotic factors decreased, while that of pro-apoptotic factors increased, possibly resulting in the increase of apoptotic cells. The latter could contribute to the functional deterioration of the bladder. We also found that different layers of bladder tissue had different apoptotic rates, which were highest in the mucosa and submucosa and lowest in the detrusor. Anoxia and active substances released by exudated WBCs could induce cell apoptosis, while inflam-
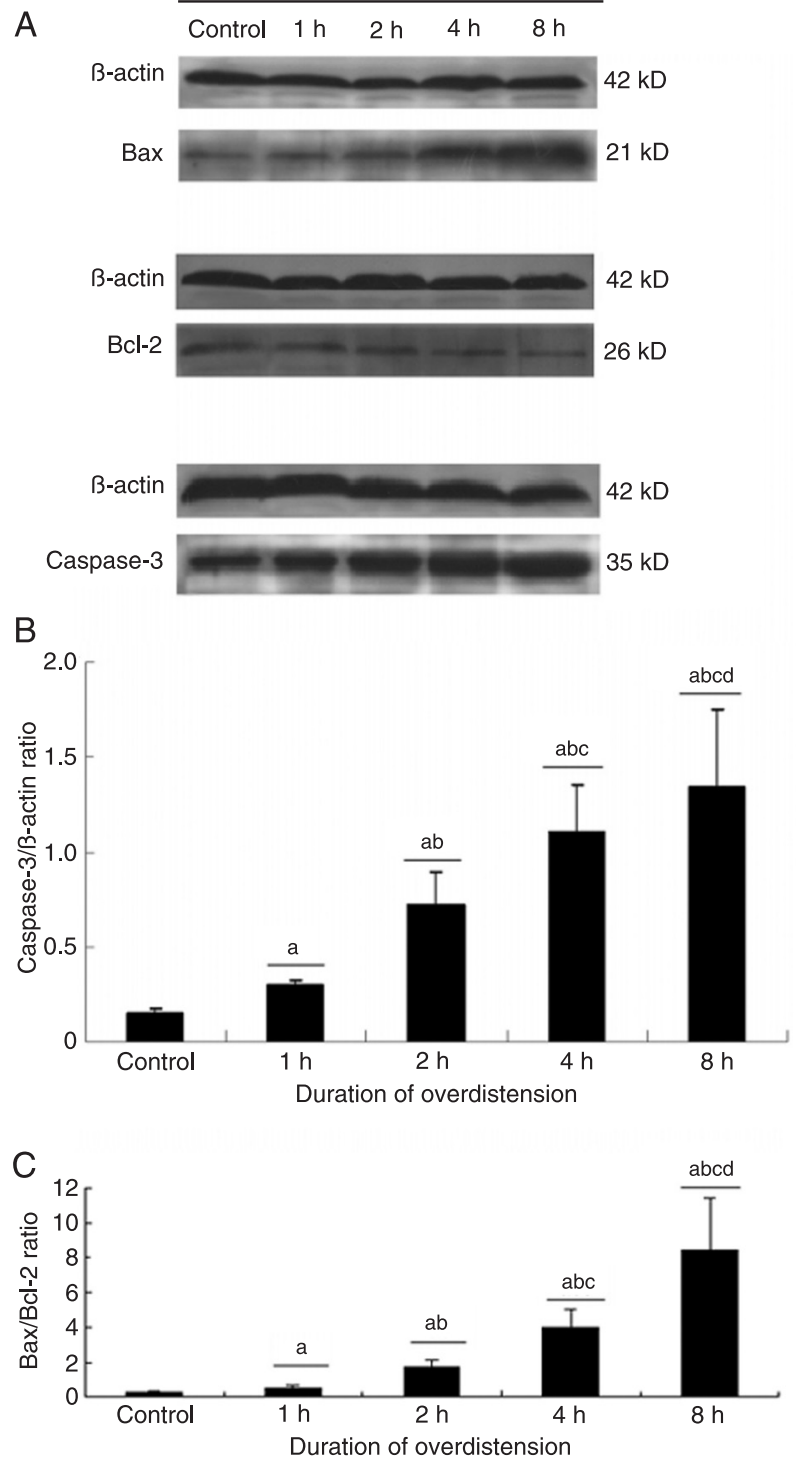

Figure 5. Effect of different durations of overdistention (DOD) on the expression of apoptosis-related proteins: $A$, Western blotting analysis of $\mathrm{Bax}, \mathrm{Bcl}-2$ and caspase- 3 , and $\beta$-actin as control. The pro-apoptotic factors Bax and caspase- 3 were up-regulated, while the anti-apoptotic factor $\mathrm{Bcl}-2$ was down-regulated with increasing DOD. $B$, Caspase $3 / \beta$-actin ratio in different $D O D$ groups, which increased significantly with increasing DOD. C, Bax/Bcl-2 ratio in different $D O D$ groups, which increased significantly with increasing DOD. All data were analyzed by ANOVA: aP $<0.05 \mathrm{vs}$ control group; bP < 0.05 vs 1 -h group; ${ }^{\mathrm{C} P}<0.05$ vs 2-h group; dP $<0.05$ vs 4 -h group.

matory exudates were most severe in the mucosal and submucosal layers, a fact that could explain the higher cell apoptosis rates in these layers. In addition, different layers of the bladder have different susceptibility to anoxia. The lowest apoptotic rates in the detrusor could be due to its 
good tolerance to anoxia.

An interesting question that many urologists may ask is "when does bladder dysfunction pass the point of no return?". Our study may provide some clues to answer this question. At the beginning of AUR, inflammatory exudates and apoptosis are limited to the mucosa and submucosa, while the contraction function of the bladder is normal. With the increase of AUR duration, inflammatory exudates and apoptosis extend to the muscle but they are still slight, while the contractile function of the bladder begins to decrease. We think that bladder dysfunction in this phase could be completely reversed because of the mild injuries to the detrusor. With the advancement of AUR, the inflammatory exudates and apoptosis become more serious in muscle and may result in irreversible impairment of the detrusor and bladder dysfunction. However, how the AUR duration affects the recovery of bladder dysfunction should be further studied.

These results remind us that we should decompress the bladder as soon as possible and shorten the duration of AUR to protect bladder function when we treat patients with AUR, although we should treat the patients with AUR on an individual basis. Patients undergoing a longer time of AUR may need a longer time to recover bladder function. Catheterization should be prolonged in such patients in order to obtain a greater success rate of trial without catheter (15). A urodynamic test before transurethral resection of the prostate should be recommended to reduce the rate of surgical failure in patients with a longer time of AUR,

\section{References}

1. Roehrborn CG, Siami P, Barkin J, Damiao R, Major-Walker $\mathrm{K}$, Nandy I, et al. The effects of combination therapy with dutasteride and tamsulosin on clinical outcomes in men with symptomatic benign prostatic hyperplasia: 4-year results from the CombAT study. Eur Urol 2010; 57: 123-131.

2. Kaplan SA, Roehrborn CG, Chapple CR, Rosen RC, Irwin $\mathrm{DE}$, Kopp Z, et al. Implications of recent epidemiology studies for the clinical management of lower urinary tract symptoms. BJU Int 2009; 103 (Suppl 3): 48-57.

3. Marberger MJ, Andersen JT, Nickel JC, Malice MP, Gabriel M, Pappas F, et al. Prostate volume and serum prostatespecific antigen as predictors of acute urinary retention. Combined experience from three large multinational placebo-controlled trials. Eur Urol 2000; 38: 563-568.

4. Choong S, Emberton M. Acute urinary retention. BJU Int 2000; 85: 186-201.

5. Lin AT, Chen KK, Yang CH, Chang LS. Mannitol facilitates rabbit urinary bladder recovery from overdistension injury. Urology 2000; 56: 702-707.

6. Tammela TL, Levin RM, Monson FC, Wein AJ, Longhurst PA. The influence of acute overdistension on rat bladder function and DNA synthesis. J Urol 1993; 150: 1533-1539.

7. Saito M, Miyagawa I. Bladder dysfunction after acute urinary retention in rats. J Urol 2001; 165: 1745-1747. who might already suffer from an irreversible impairment of detrusor function (16).

Certainly, bladder overdistention is not equal to AUR, and clinical circumstances are more complicated than animal experiments. AUR usually is secondary to bladder outlet obstruction or detrusor dysfunction $(17,18)$. The function and structure of the bladder may already have changed before AUR. In addition, other factors such as age and physical condition also contribute to the severity of impairment and the recovery of bladder function after AUR $(19,20)$.

DOD plays an important role in functional and structural impairment of the bladder. Different DOD result in different severities of impairment of the rat bladder. With increasing duration, the inflammation and the injuries to ultrastructures of the bladder are more serious and the apoptotic rates and pro-apoptotic factors are up-regulated, while antiapoptotic factors are down-regulated, all of which could contribute to more serious impairment of bladder function and structure.

\section{Acknowledgments}

We are very grateful to Mrs. Li Wang for meticulous technical assistance with transmission electron microscopy, and to Dr. Guo-hua Lin for his kind supervision of the contractile assay of muscle strips. Research supported by grants from the Department of Education of Zhejiang Province (\#491010+G21078).
8. Greenland JE, Brading AF. The effect of bladder outflow obstruction on detrusor blood flow changes during the voiding cycle in conscious pigs. J Urol 2001; 165: 245-248.

9. Gabella G, Uvelius B. Structural changes in the rat bladder after acute outlet obstruction. Scand J Urol Nephrol Suppl 1999; 201: 32-37.

10. Abrams P, Cardozo L, Fall M, Griffiths D, Rosier P, Ulmsten $U$, et al. The standardisation of terminology of lower urinary tract function: report from the Standardisation Sub-committee of the International Continence Society. Neurourol Urodyn 2002; 21: 167-178.

11. Saito M, Shimizu S, Kinoshita Y, Satoh I, Shomori K, Dimitriadis $\mathrm{F}$, et al. Bladder dysfunction after acute urinary retention in the rats: a novel over active bladder model. Mol Cell Biochem 2010; 333: 109-114.

12. Hong SK, Son H, Kim SW, Oh SJ, Choi H. Effect of glycine on recovery of bladder smooth muscle contractility after acute urinary retention in rats. BJU Int 2005; 96: 14031408.

13. de Souza GM, Costa WS, Bruschini H, Sampaio FJ. Morphological analysis of the acute effects of overdistension on the extracellular matrix of the rat urinary bladder wall. Ann Anat 2004; 186: 55-59.

14. Thorne MB, Geraci SA. Acute urinary retention in elderly 
men. Am J Med 2009; 122: 815-819.

15. Desgrandchamps F, de laTaille A, Doublet JD. The management of acute urinary retention in France: a cross-sectional survey in 2618 men with benign prostatic hyperplasia. BJU Int 2006; 97: 727-733.

16. Dubey D, Kumar A, Kapoor R, Srivastava A, Mandhani A. Acute urinary retention: defining the need and timing for pressure-flow studies. BJU Int 2001; 88: 178-182.

17. Thomas AW, Cannon A, Bartlett E, Ellis-Jones J, Abrams P. The natural history of lower urinary tract dysfunction in men: minimum 10-year urodynamic follow-up of untreated bladder outlet obstruction. BJU Int 2005; 96: 1301-1306.

18. Verhamme KM, Sturkenboom MC, Stricker BH, Bosch R. Drug-induced urinary retention: incidence, management and prevention. Drug Saf 2008; 31: 373-388.

19. Selius BA, Subedi R. Urinary retention in adults: diagnosis and initial management. Am Fam Physician 2008; 77: 643650.

20. Daly P, Connolly S, Rogers E, Sweeney P. Management outcome of acute urinary retention: model of prediction. Urol Int 2009; 83: 39-43. 